\title{
The impact of dental anxiety on daily living
}

\author{
S. M. Cohen, ' J. Fiske, ${ }^{2}$ and J. T. Newton, ${ }^{3}$
}

\begin{abstract}
Aim To explore the impact of dental anxiety on daily living. Method Twenty people attending a dental sedation clinic completed the Modified Dental Anxiety Scale, and were interviewed privately using a reflexive, in-depth technique. All interviews were audio-tape recorded and transcribed. The transcripts were analysed to identify the impact of dental anxiety upon the participants' daily lives. Twenty-five per cent of the qualitative data was reviewed by an independent researcher to ensure the reliability of the analysis.
\end{abstract}

Results The mean age of participants was 41 years (range 23 to 60). The mean MDAS score was 21.5 (range 14 to 25). Five main impacts of dental anxiety were identified: physiological; cognitive; behavioural; health; and social. Subsumed under these broad categories were: the fright response; a vast array of negative thoughts, feelings and fears; avoidance behaviour and behaviours related to eating, oral hygiene, and self-medication; and other manifestations of anxiety in the dental environment including muscular tension, crying and aggression were all identified. Dental anxiety was also found to disturb sleep and to have a profound affect socially, interfering with work and personal relationships.

Conclusion The impact that dental anxiety can have on people's lives is wide-ranging and dynamic.

\begin{abstract}
The prevalence of dental anxiety is high. The 1988 United 1 Kingdom Adult Dental Health Survey ${ }^{1}$ and other studies ${ }^{2,3}$ indicate that about a third of the UK adult dentate population are dentally anxious. Dental anxiety has also been identified as a significant barrier towards the receipt of dental care, particularly as the result of avoidance. ${ }^{4,5}$ Dental anxiety does not solely affect patients, general dental practitioners identify treating nervous patients as a major source of stress. ${ }^{6}$

There is little information on the direct impact that dental anxiety has on people's lives. A number of studies have indicated that people who are dentally anxious experience a greater amount of dental disease. ${ }^{7-10}$ Additional evidence that dental anxiety is detrimental to oral health comes from the 1988 United Kingdom Adult Dental Health Survey. ${ }^{1}$ People who selected a fear-related statement as the most important barrier to dental care, had more missing teeth and fewer filled teeth than those who had no strong association with any of the potential barriers to dental attendance.
\end{abstract}

\footnotetext{
$1^{*}$ Dental Officer, Community Dental Service, Riverside NHS Trust; ${ }^{2}$ Senior Lecturer, Honorary Consultant in Special Care Dentistry, at Guy's, King's and St Thomas' Dental Institute, King's College London; ${ }^{3}$ Lecturer in Psychology in Relation to Dentistry at Guy's, King's and St Thomas' Dental Institute, King's College London

${ }^{*}$ Correspondence to: Dr Janice Fiske, Department of Sedation and Special Care Dentistry, Floor 26, Guy's Tower, London SE1 9RT email: janice.fiske@kcl.ac.uk

REFEREED PAPER

Received 03.02.00; Accepted 09.06.00

(c) British Dental Journal 2000; 189: 385-390
}

The development and use of sociodental indicators by several researchers ${ }^{11}$ has demonstrated the psychosocial impact of dental disease. However, relatively few studies appear to have investigated the direct impact of dental anxiety on the quality of life. In one study by Berggren, ${ }^{12}$ a proportion of patients who had been referred to a dental anxiety clinic indicated that their dental fear caused problems either with social activities (meeting friends, eating out) $(52 \%)$, going on holiday (46\%) or with family relationships (41\%). Less affected were work (37\%), leisure activities (hobbies, sports) $(25 \%)$, and domestic duties (eg cleaning or cooking) (14\%). The findings of this research are limited by the simple 'yes' or 'no' responses to items, which did not allow respondents to indicate the extent and quality of the impact which they identified.

The aim of the current study is to explore the impact of dental anxiety on daily living. A qualitative methodology is adopted to allow identification of the full range of impacts identified by participants. Such an approach has been used previously in dental settings to examine a range of topics, including the emotional effects of tooth loss, ${ }^{13}$ the impact of loss of part of the face ${ }^{14}$ and the stress in dental practice. ${ }^{15}$

\section{Method}

Twenty adult subjects were recruited from patients attending the Dental Sedation Unit in the Department of Sedation and Special Care Dentistry at Guy's, King's and St Thomas' Dental Institute of King's College London (GKT) for treatment with sedation. All had been referred by a dental practitioner for management of their dental anxiety. They were either new patients attending for assessment or existing patients attending for review and were expected to have high levels of dental anxiety. Potential subjects were invited to participate in the study. The purpose and method of the study were explained, patients were assured of confidentiality and anonymity. A mutually convenient interview appointment was arranged and written informed consent was obtained at the time of the appointment.

The study involved quantification of the study sample's levels of dental anxiety and qualitative exploration of the impact that the anxiety had on their lives.

The Modified Dental Anxiety Scale: The Modified Dental Anxiety Scale (MDAS) ${ }^{16}$ was used to quantify the participants' levels of dental anxiety and to confirm their expected levels of high anxiety concerning dental treatment. A MDAS score of 19 and above indicates a strong likelihood of the respondent being dentally phobic. ${ }^{16}$ Following completion of this questionnaire, the patient's age, sex and MDAS score were noted.

Depth interviews: Depth interviews, conducted in private, were used to study the impact of dental anxiety on the study population's daily living. All the interviews were audio-tape recorded and conducted by one researcher. They were subsequently transcribed. A list of interview topics was used as a check-list for the interviews to ensure that all areas of interest to the researcher were covered. This topic list was initially developed from a review of the literature and the researchers' experiences of the effects of dental disease and anxiety on the quality of life; and reviewed in the light of ten pilot interviews to provide a definitive topic list. This included: the history of 
the dental anxiety; strategies for coping with dental anxiety; feelings and concerns about visiting the dentist; the ability to talk about dental anxiety; the impact of dental anxiety on oral self-care; the impact of dental anxiety on day-to-day living; the impact of dental anxiety on eating; and the impact of dental anxiety on social interaction/communication.

The interviews were reflexive, the interviewer explored topics raised by the participants, responding to the topics raised by the participant rather than strictly following a pre-defined interview schedule.

Data analysis: The MDAS scores and demographic data are presented numerically. The qualitative data were analysed by one researcher (SC) scrutinising the transcriptions to identify the themes that arose relating dental anxiety to aspects of daily living. Five interview transcripts (chosen randomly, 'numerically' representing 25\% of the qualitative data) were reviewed by an independent researcher (JTN) to minimise interpreter bias. Inter-rater reliability between the researchers was measured using a coding system consisting of the themes identified for the impact of dental anxiety.

\section{Results}

Demography: The sample comprised 14 women and 6 men. Their ages ranged from 23 to 60 years with a mean of 41 years, and a median age of 39.5 years.

MDAS scores: These ranged from 14 to 25 with a mean and median of 21.5 and 22 for men and women respectively. Fifteen people ( $75 \%$ of the sample) scored 19 and above. Of the remaining five people ( $25 \%$ of the sample), four scored 18 and one person scored 14.

Qualitative data: The duration of the interviews ranged from 30 to 86 minutes with a mean of 51 minutes. Seventy-five per cent of the interviews ranged from 35 to 65 minutes with a mean of 49 minutes. Agreement between the researchers was $100 \%$ for the identification of emerging themes and ranged between $83 \%$ and $96 \%$ for the coding of impacts within the themes.

Five broad themes were identified relating to the impact of dental anxiety each having sub-themes. The themes and sub-themes identified are listed in Figure 1.

Three of the themes - physiological, cognitive and behavioural — are illustrative of the three components of anxiety. ${ }^{17}$ They were recognised as having an impact, not only on the day of a dental appointment (including the following 24 hours), but also, spatially and temporally outside the dental environment. The 'health' theme illustrates the impact that dental anxiety has on oral health and sleep. While the 'social' theme presents the impact dental anxiety has on work and relationships. In the tradition of qualitative research the results are illustrated with verbatim quotes.

\section{Physiological impacts}

Fright response: The majority of people reported experiencing physiological symptoms of fear such as dry mouth, sweatiness, increased heart rate, etc. These symptoms occurred prior to (most usually on the day or night before), and on, the day of a dental appointment. They occurred most frequently in the waiting room or the dental surgery, though similar feelings were also expressed while telephoning to obtain help finding a dentist.

'... and when I was phoning I used to get, my heart used to start racing, I used to go hot and cold, used to hyperventilating, that was just, that was just purely phoning round the Health Authority and asking for people that they recommended.'

Additionally, a few people expressed feeling exhausted after a dental appointment to an extent that they could not carry out normal daily living activities:

'... it's like running the marathon or having a fight with Mike Tyson. I'm totally exhausted, you know, like I come back, I had no treatment yesterday, but when I got back here, I had to take a hot shower and

\section{Fig. I Themes and sub-themes identified from interviews}

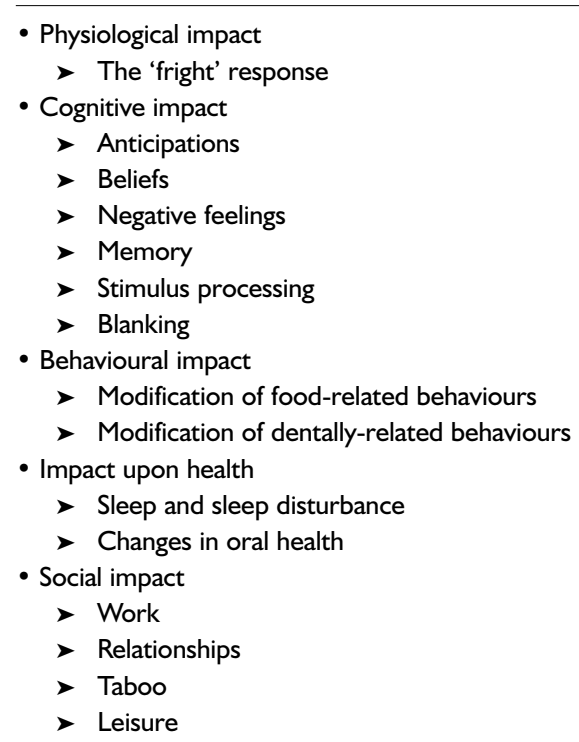

I had to just sit here and had to have a drink, and I just almost couldn't do anything. I was totally and utterly shattered and I hadn't even had anything done.'

\section{Cognitive impacts}

Dental anxiety is commonly manifest as changes in thought patterns or content. A great number of these were expressed by participants. They were categorised into six groups: anticipations; beliefs; negative feelings; memory; stimulus processing; and blanking.

Anticipations: These included fear of losing teeth; fear of death; ruminating on what was going to happen during time spent in the waiting room; and catastrophic thoughts about what might happen.

'... I think the trouble is, in waiting rooms, you have too much time to ponder, to think about what's going to happen to you, you know. I mean, sometimes you're sitting out there for twenty minutes or more, and that's too long. For somebody like me it is.... to sit in that waiting room, it's like torture to me.'

Beliefs: This category included statements in which the participants expressed their views, and their opinion of the views of others. Often beliefs referred to the value placed on particular states and experiences. People with dental anxiety felt they were perceived by other people to be stupid, silly or weak; and that other people lacked an appreciation or understanding of their anxiety. As a result they sometimes felt stigmatised. Some people considered extraction of all of their remaining natural teeth, believing this once and for all solution to be a better prospect than that of having to visit the dentist repeatedly:

'If I had my way, I'd have them [my teeth] all out and have false teeth. Yeah, because I can't get toothache. They're easy to keep clean.'

Far fewer 'beliefs' were expressed (and by fewer people) in relation to the day of a dental appointment. What was expressed, though, was a preference not to have to wait and to have the dental treatment completed as soon as possible:

'...I don't like the waiting around. That's why when they give you an appointment and a time, you better be ready to see me or sometimes you ain't seeing me because I'll get up and leave.'

Negative feelings: These included vulnerability, a loss of control, and low self-esteem. The first two states were more often experienced during the time spent in the dental chair:

'... you're out of control, and you're in a chair, you're virtually, you know, laid back horizontal, lights and things and things in your mouth and, you know, you're totally helpless.'

Memory: A few people were plagued by the memory of previous traumatic dental experiences which were vividly replayed: 


\section{RESEARCH behavioural dentistry}

'I have that picture of when I was a kid and it just won't come out of my head and that's it.'

Stimulus processing: This refers to hypersensitivity to dental stimuli and/or expectation of pain. It was mentioned by a majority of participants and was the impact most often voiced in relation to the day of the dental appointment. In general, people disliked the sight and sound of dental equipment, the smell of the dental environment and the vibration of the drill. They had an expectation of pain, being hurt and of choking or gagging during treatment:

'... my main problem is actually having someone that I can see working on my teeth. Seeing horrible, you know, things like drills, hearing the noises, smelling the smells, don't like it.'

Blanking: The technique of blanking the mind in the dental chair was a successful coping strategy for some people. It was not widely used and some people acknowledged the difficulty of blanking whilst feeling extremely anxious:

'...my brain goes at a thousand miles an hour, everything from trying not to thinking about it to thinking about it, to aspects of it as to will he slip, won't he slip, what's he gonna say, is, I cannot even begin to describe the number of thoughts that go through my head. Sometimes I would deliberately try and think of anything but.'

\section{Behavioural impacts}

Modification of food-related behaviours: These were expressed by the majority of people and included avoiding eating certain foods, particularly hard foods (because of a reduced ability to chew or to prevent a dental problem) or cold foods (caused by sensitivity).

Modification of dentally-related behaviours: These were related to oral hygiene and avoidance of dental treatment and the dental setting. Most participants reported that dental anxiety had a positive impact on their oral hygiene practices. Their motivation was to avoid the need to have dental treatment by preventing dental disease. To this end they were prepared to purchase what they felt were the best aids to oral hygiene:

'I do everything you're supposed to do except visit the dentist. I change my toothbrush regularly, I always buy a good make of toothbrush and toothpaste and, um, I clean my teeth regularly and I use a mouthwash, and, um, make sure that I brush not just my teeth, but my gums as well. Yeah. It's all avoidance.'

Avoidance of the dental environment was expressed by all the interviewees. It is characterised by failed appointments, infrequent attendance, and several years of non-attendance:

'That's why I don't go to the dentist regularly unless you call about every ten years regularly'

Avoidance behaviour may persist despite the presence of acute pain:

'To me, it [avoiding dental visits] makes absolute sense because no matter how much pain I've got in my mouth, it's nothing like the fear and loathing that I have in my mind.'

However, in some cases, attendance can eventually no longer be avoided due to pain or a 'major problem':

'I've put up with about six years [of pain] on and off, that I thought, you know, it's about time. So really, the only reason why I'm, I've come is because I can't put up with the pain anymore.'

Attendance is often a last resort, and a few participants self-medicate to avoid going to the dentist. Self-medication included the use of antibiotics and over the counter analgesics. Occasionally this leads to unsafe behaviour:

'... sometimes I get used to the paracetamol and I used to take like loads at a time, then I overdose on paracetamol and, but the pain goes.'

For some people, avoidance behaviour extended to a refusal to look at media, TV or film portrayals of dentistry:

'.....saw "Marathon Man" a little while back. I've not seen it and then, er there was, er, some horrible Nazi dentist in it, and I had to switch the film off. I just couldn't watch it. Didn't care what happened in the end.'

Other aberrant behaviours included crying and aggression particularly in the dental practice. Crying was common:
'... and I'll always end up in tears, crying all over the place and almost like a small child, you can't control your emotions.'

A few participants (both male and female) admitted being physically aggressive towards the dentist in the past:

'I hit him [the dentist] ... and I took him straight off his feet and I said, 'You are just sticking needles in my mouth and you are not gonna do it again', and I walked out.'

\section{Health impacts}

Sleep and sleep disturbances: These formed the main thrust of the impact of dental anxiety on health. An inability to sleep the night prior to a dental appointment was commonplace. Additionally, though less frequently, sleep was disturbed by dreams or nightmares related to dental fears at times remote from a dental appointment:

'I'm better now because the bad dreams or the 'sleeplessness' night is the night before. I mean it used to be a few days or even a week up to an appointment.'

Changes in oral health: Very few people indicated that their oral health was worse as a result of their dental anxiety:

'I put up with this swell, swelling for weeks because I didn't want to go and have anything done about it.'

\section{Social impact}

Dental anxiety affected people's lives in the workplace and their personal relationships.

Work: At work the impact was on both social interactions (concealing feelings or seeking support) and work performance. A small number of people regretted revealing their dental anxiety to work colleagues. In one case an admission of dental anxiety was perceived as undermining managerial authority by portraying weakness:

'...I won't be revealing that sort of information [about my dental fears] to again in future, but that's the only, that's the only, because, you know, when I do my job, I'm like, I'm a manager so I come across in a certain way and it's like, I think with that particular person, she may have seen that as a weakness.'

Others sought emotional support from work colleagues:

'I work in an office where we're all very close to each other anyway... so I'm always like 'Oh, I've got to go to the dentist tomorrow'. Maybe I'm seeking sympathy or something. I don't really know but everybody, everybody I know, knows I'm going.'

Some people recognised that their mood and behaviour changed as a dental appointment approached and they avoided social contact with colleagues at this time:

'... I'm very, very aware that I've got the, an appointment coming $u p$, um, yeah, and I start getting nervous about it ... I'm very aware that I can be, um, very snappy with other people, so I try, and kind of step back and keep myself to myself in work.'

The adverse effect severe dental anxiety has on oral health can have an impact upon a person's ability to work or gain promotion:

'Um, I've had a couple of times when I've had dental pain and I've had to basically stop [working]. Obviously the anxiety caused the pain because I didn't have the [dental] work done. ... In some respects it's also affected my work prospects. You don't look good on an interview if you don't speak properly, and if you don't speak properly because you don't want your teeth to be seen.'

The significant impact on work that a dental appointment has in terms of time required to recover from the experience (particularly if it involved intra-venous sedation) was emphasised:

'Sometimes it's daunting for me to think 'Oh, yeah I've got to take a whole day to go to the dentist, um, rather than some people just taking a couple of hours, and that's that', ... so I find myself other than working late, or, I'll work one day a weekend or something to try and catch up. Sometimes that's challenging, ...'

Relationships: Severe dental anxiety can have a detrimental effect on a developing close personal relationships: 
'Yeah, and socially it is damming. I have actually walked out of a dinner once because I actually felt so embarrassed, and the woman I was with had beautiful white teeth. Straight. I was embarrassed, and I walked out... It killed it [the relationship] stone dead. I, I, I literally, I phoned her up the next day and that was it.'

Existing close personal and family relationships may also be strained by dental anxiety, particularly approaching a dental appointment:

'I mean, the bloke who I'm dating now, he brought me last week for my appointment and once again, had to stop the car to, and he kept saying to me 'But you're only going for a check-up, just to see what', and I said 'Yeah, but this is what I'm like' and he found it a bit hard. He couldn't believe it that I was like that.'

People with severe dental anxiety are less likely to accompany an adult or a child (including their own children) to the dental surgery:

'No, there's no way I'd take a child, absolutely no way whatsoever, and if I, if I went with an adult, I wouldn't probably, probably walk into the surgery, I'd wait outside ... outside in the street.'

Most parents also perceived that their dental anxiety could be imparted to their children. They admitted finding it difficult to conceal their anxiety:

'But I've had to appear brave since I've had my kids, because I have to take the children. ... You have to take the kids and you can't show the children that you're scared of the dentist, otherwise they're not gonna go.'

Taboo: A majority of people indicated that they would prefer not to discuss their dental anxiety or the topic of dentistry with other people:

'It's not the sort of thing I think they [people] would want to know. ... It's not like an everyday topic you can discuss with someone, if you know what I mean, you can't bring it up in a conversation, not like boyfriends or marriage or children, and things like that. It's just something you don't think people really wanna know or interested in.'

Leisure: A minority of participants mentioned instances of dental anxiety affecting their leisure pursuits:

'I mean I, I did pull out of a fishing competition because I was in dental pain. I mean I had a toothache, and I started fishing a five hour match, and within two hours I had to pack everything up, and I was in absolute agony...'

\section{Discussion}

\section{Sampling bias}

As the study sample was self-selected, it is arguable whether or not it can be considered representative of a group of severely dentally anxious people in the general population. The participants form a convenience sample. ${ }^{18}$ It is acknowledged that patients attending the Department of Sedation and Special Care Dentistry at GKT for dental treatment with sedation are likely to have higher levels of dental anxiety than those attending general dental practitioners. Using the qualitative method, the findings confirm previous suggestions in the literature.

Although the impacts related to dental anxiety will be discussed individually, they rarely exist as static or separate entities. Freeman describes barriers to dental care as 'resistances' which 'ebb and flow in accordance with the patient's feelings, worries and anxieties, on the one hand, and the desire for treatment on the other hand. ${ }^{19}$ This description endorses the dynamic nature of dental anxiety illustrated by the current research. The MDAS scores of the study sample indicated that $75 \%$ of the group were dentally phobic according to Humphris et al.'s criteria. ${ }^{16}$ Although the MDAS scores of the remaining $25 \%$ of the group indicated they were not dentally phobic at the time of interview, they had a history of dental phobia prior to attending for dental treatment with sedation. The one person (5\%) reporting the lowest MDAS score is unlikely to distort the results, because qualitative research reports impacts rather than numbers.

\section{The range of impact of dental anxiety}

The results of this study illustrate the very wide range of impact dental anxiety can have on people's lives. Dental anxiety affected the participant's physiological reactions; psychological state; daily living activities such as eating and work performance; and relationships - both socially and at work. Thus, the impact of dental anxiety is broader than the 'psychosocial' impact reported by Berggren. ${ }^{12}$

The participants in the present study had all demonstrated marked levels of dental anxiety, sufficient to require referral for treatment with sedation. As such they would be classified as dentally phobic. This impact of this level of dental anxiety is high and effects the individual not only on the day of the appointment but at times distant from the appointment. This is a useful distinction for the general dental practitioner attempting to decide whether a patient's level of anxiety is sufficient to warrant referral for specialist care. Where the impact of anxiety is wide ranging (affecting relationships, work and general health both at the time of the appointment and at other times) and severe, specialist care is recommended.

The finding that dental anxiety has a marked impact upon daily living does not imply any causal mechanism for the development and maintenance of such fear. Both behavioural and psychodynamic models of the development and maintenance of dental anxiety have been proposed. ${ }^{20,21}$

\section{Physiological impact}

The physiological signs and symptoms people expressed are similar to the somatic symptoms of anxiety described by Rees et al. ${ }^{22}$ and the signs of dental anxiety listed by Speirs. ${ }^{23}$ Feeling exhausted after an appointment is an additional finding. This is in accordance with the physiological reaction of fight or flight which animals (including humans) experience when faced with perceived danger. Thus, it is not surprising that more people reported physiological signs and symptoms occurred on the day of a dental appointment than on the day or night before.

\section{Cognitive impact}

Amongst the cognitive impact, anticipation of being reprimanded by the dentist is recognised as a concern. ${ }^{4}$ This is grounded in the reality of 'victim blaming' which has been reported amongst health professionals, including dentists. Jacob and Plamping pointed out that: ${ }^{24}$ 'Victim blaming is counterproductive and has the effect of returning the patient to the pre-contemplation and contemplation stages of behaviour change, where she may question if she wants to change anyway'. Thus, anxiety and avoidance behaviour may be mutually reinforced if victim blaming is experienced in the dental environment.

Fear of the unknown or the uncertainty of dental treatment, with particular reference to the expectation of pain, is well recognised as a contributory factor to the aetiology of dental anxiety. ${ }^{25}$ However, fear of death (which is recognised as one of the cognitive symptoms of anxiety $)^{22}$ has not been related to dental anxiety previously. In the current study, the immediate period of waiting for dental treatment was commonly cited as being particularly anxiety-provoking, as this increased the time available to think about what was going to happen and to ruminate on worst outcomes. It has been suggested by the lay public that reducing patient waiting time would help reduce anxiety. ${ }^{4}$

Another fear vocalised in the study was the belief of being perceived as stupid, silly or weak. Beliefs about other people's lack of appreciation or understanding of dental anxiety sometimes resulted in feeling stigmatised. This endorses the findings of other researchers ${ }^{26}$ and may contribute to the participants' opinion that their dental anxiety is a taboo subject.

Negative feelings expressed by the study group included feeling vulnerable, losing control, and low self-esteem. Participants cited a feeling of vulnerability and loss of control in the dental environ- 
ment in general, and in the dental chair in particular. These are not well established findings relating to dental anxiety, although there is a small evidence base for them. ${ }^{4,16}$ Providing control has been shown to be effective in reducing anxiety before a dental appointment by informing new patients in a short leaflet that they could stop their dentist during treatment. ${ }^{27}$ In practice many dentists give control to the patient using a signalling system, which is particularly useful with children and anxious adults.

Memories and vivid recollections of previous, traumatic, dental experiences were reported by only a few people in the current study. However, the role of memory should not be under-estimated in the aetiology and perpetuation of dental anxiety since it is implicit in the learning process. ${ }^{28}$ These memories or flashbacks serve to reinforce the dental anxiety and repudiate later good (or not so bad) dental experiences. Indeed, a criticism which has been levied at the pharmacological control of dental anxiety is that 'the amnesic effects of some sedative drugs can make it difficult for patients to learn to appreciate that treatment is not as daunting as they expect.'. ${ }^{29}$

\section{Behavioural impact}

The avoidance of eating certain foods (particularly hard or chewy ones perceived as being potentially damaging to teeth) was commonly expressed by the study group who often linked this behaviour to their dental anxiety. It is debatable whether this is due to oral health status (such as pain from a carious lesion), rather than a direct consequence of dental anxiety (such as fear that eating something hard will result in a broken tooth necessitating dental treatment). However, the literature supports the view that dentally anxious people are more likely to have poor, or poorer, oral health status compared with people who are less, or not, dentally anxious. ${ }^{1,7-10}$ It has been suggested that: 'the dental phobic is caught in a vicious cycle, where fear, pain, and feelings of guilt and inferiority may prevent good oral hygiene and treatment.' ${ }^{\prime}$ Conversely, in this study people commonly reported that dental anxiety had a positive impact on their oral hygiene practices. Their motivation was to avoid the need to undergo dental treatment by preventing dental disease. They not only put extra effort into improving hygiene but also spent extra money in buying the 'best' products.

There is a body of evidence to support the impact of dental disease on the quality of life. ${ }^{11}$ Such research may include an indirect measure of the relationship between dental anxiety and quality of life, given that the avoidance of dental care can result in poorer oral health. In this study, avoidance of the dental environment was identified as the most common impact of dental anxiety. Avoidance behaviour is well recognised as being a response to dental anxiety. ${ }^{5}$ A consequence of avoidance means that chronic and even acute pain may have to be endured. Segal reported that $15 \%$ of people attending an emergency clinic had been in constant pain for a month or more. ${ }^{30}$ Eventually attendance may be triggered, when the frequent experience or severity of pain can no longer be borne, or, a major problem is experienced. However, people may self-medicate to continue to avoid going to the dentist. Commonly, they may self-administer antibiotics and/or 'over-the-counter' analgesics/ painkillers. Indiscriminate use of antibiotics can result in resistance, hypersensitivity and even anaphylaxis. Inappropriate topical administration of aspirin can cause oral ulceration, and potentially fatal overdose with paracetamol is recognised. Thus, avoidance could have far-reaching consequences.

The media portrayal of negative images of dentistry reinforces the stereotype of the stern dentist inflicting pain on an often anxious patient. ${ }^{31}$ In the current study, some respondents described their avoidance of media, TV or film portrayals of dentistry. They also stated the effect it had on them if this type of dental exposure was experienced unexpectedly. Finch et al. suggested that a promotional campaign was needed to dispel the negative image of pain and discomfort that people tend to associate with dentistry. ${ }^{4}$ The portrayal of positive dental images in TV soap dramas or 'sit-coms' might also be helpful to redress the balance.

Although not a well reported finding in the dental literature, this study illustrates that aggressive behaviour may, on occasions, be due to dental anxiety. Such attacks are acknowledged in an advisory publication from the British Dental Association: ${ }^{32}$ 'Physical assaults take place during treatment and can take the form of bites on fingers, hands, arms or other parts of the body by frightened patients, often children.'. Paradoxically, this seemingly disproportionate reaction to dental anxiety may occur as a result of the individual feeling assaulted and vulnerable as their personal space is invaded by the dentist. However, it should be remembered that 'fight' is a basic physiological reaction to fear.

\section{Health impact}

Within the current study, the main impact of dental anxiety on health was as a consequence of sleep disturbance, either as an inability to sleep or as the result of dreams or nightmares. Although sleep disturbance caused by dental and facial pain has been reported, ${ }^{33}$ sleep disturbance related to dental anxiety does not appear to be an established finding.

Fewer people than the researchers expected indicated that their oral health was worse as a result of their dental anxiety. This is surprising in view of the evidence in the literature relating anxiety to compromised oral health. ${ }^{1,7-10}$ However, this finding assumes a narrow, clinical, definition of oral health status. Oral health has been defined as: '.. a standard of health of the oral and related tissues which enables an individual to eat, speak or socialise without active disease, discomfort or embarrassment and which contributes to general well-being. ${ }^{34}$ By this definition, and the findings of the present study, dental anxiety severely compromises 'oral health' since it has been found to impact upon social and psychological well being. Clinical oral health status was not measured in this study, therefore, it was not possible to determine objectively the direct impact of dental anxiety on clinical indices. Such a relationship could be quantitatively investigated in future longitudinal studies.

The impact of dental anxiety on oral health may have further consequences for the patient. Increased use of general medical services for prescription of antibiotics, and use of analgesics have been reported amongst dental phobics. ${ }^{21}$

\section{Social impact}

Although the social impact of dental disease is established, its relation to dental anxiety is not well documented. This study demonstrated clearly that dental anxiety can affect people's lives at work and also their personal relationships. At work it affected relationships through the need to: conceal the perceived weakness of anxiety; avoid colleagues because of irritability associated with dental appointments; or seek peer group support. Oral pain and infection as a consequence of the effects of dental anxiety on oral health interfered with the ability to work. Similarly, poor dental appearance affected career prospects by creating a negative impression at interview and lowering both self-esteem and self-confidence.

The present study demonstrated dental anxiety can have a detrimental effect on developing close relationships. Similar findings have been reported for individuals with facial disfigurement and for individuals who experience tooth loss. ${ }^{13,35}$ Established personal relationships can also be strained. People recounted becoming irritable or agitated around dental appointments and perceived partners to lack understanding or tolerance of their anxiety.

Parents were unable to accompany their children to the dentist because of their own anxiety and to avoid transferring their fear. The relationship between parental and child dental anxiety is well recog- 
nised. Although the presence of the parent in the dental surgery is advocated, the exception is where 'the mother is too anxious and herself too frightened of dental treatment', as this will have a negative effect on the 'treatment alliance' between the dentist and the child patient. ${ }^{36}$

\section{Taboo}

In many instances the study population felt unable to discuss their dental anxiety with other people. It is difficult to identify the reason for this taboo as the participants were not able to explain it other than in terms of 'something people do not talk about'. Interestingly, the emotional effects of tooth loss have also been identified as taboo, with some people finding it easier to express their feelings about cancer. ${ }^{13}$ It may be that people find discussing anxiety emotionally upsetting as it rehearses their bad experiences. In contrast, in the environment of a support group, it was considered the norm to express fear of the dentist and to discuss the causes. ${ }^{37}$ A major contribution to the success of the group was this 'public' acknowledgement of the anxiety itself. Attendance at a group meeting dissipated dental anxiety and promoted feelings of empathy and confidence. Members of the support group soon accepted and completed a course of dental treatment, with few failed attendances. It has been suggested that wider availability of such groups could help to reduce dental anxiety in non-attending adults and encourage treatment uptake. ${ }^{37}$

\section{Conclusions}

The impact that dental anxiety can have on people's lives is wideranging and dynamic. Physiological impacts include signs and symptoms of the fright response and feelings of exhaustion after a dental appointment, while, cognitive impacts include an array of negative thoughts, beliefs and fears. Behavioural impacts include not only avoidance, but other behaviours related to eating, oral hygiene, self-medication, crying and aggression. The main impact of dental anxiety on general health was as a consequence of sleep disturbance. Additionally, dental anxiety was found to affect social interactions and performance at work; and that, linked to feelings of low self-esteem and self-confidence, dental anxiety can also have a profound effect on both established and new personal relationships.

1 Todd J E, Lader D. Adult Dental Health 1988 United Kingdom. London: HMSO, 1991.

2 Lindsay S J E, Humphris G, Barnby G J. Expectations and preferences for routine dentistry in anxious adult patients. Br Dent J 1987; 163:120-124.

3 Mellor A C. Dental anxiety and attendance in the North-west of England. J Dent 1992; 20: 207-210.

4 Finch H, Keegan J, Ward K, Sanyal-Sen B. Barriers to the receipt of dental care. A qualitative research study. Social and Community Planning Research, 35 Northampton Square, London EC1V 0AX, 1988.

5 Kleinknecht R A, Bernstein D A. The assessment of dental fear. Behavior Therapy 1978; 9: 626-634.

6 Cooper C L, Watts J, Kelly M. Job satisfaction, mental health and job stressors among general dental practitioners in the UK. Br Dent J 1987; 162: 77-81.

7 Hällström T, Halling A. Prevalence of dentistry phobia and its relation to missing teeth, alveolar bone loss and dental care habits in an urban community sample. Acta Psychiatrica Scand 1984; 70: 438-446.
8 Berggren U, Meynert G. Dental fear and avoidance: causes, symptoms, and consequences. J Am Dent Assoc 1984; 109: 247-251.

9 Locker D, Liddell A. Clinical correlates of dental anxiety among older adults. Community Dent Oral Epidemiol 1992; 20: 372-375.

10 Hakeberg M, Berggren U, Gröndahl H-G. A radiographic study of dental health in adult patients with dental anxiety. Community Dent Oral Epidemiol 1993; 21: 27-30.

11 Slade G. Measuring Oral Health and Quality of Life. Chapel Hill: University of North Carolina, Dental Ecology, 1997.

12 Berggren U. Psychosocial effects associated with dental fear in adult dental patients with avoidance behaviours. Psychology Health 1993; 8: 185-196.

13 Fiske J, Davis D M, Frances C, Gelbier S. The emotional effects of tooth loss in edentulous people. Br Dent J 1998; 184: 90-93.

14 Newton T, Fiske J, Foote O, Frances C, Loh I M, Radford D R. A preliminary study of the impact of the loss of part of the face and its prosthetic restoration. J Prosthet Dent 1999; 82: 585-590.

15 Newton T, Gibbons D E. Stress in dental practice: A qualitative comparison of dentists working within the NHS and those working within an independent capitation scheme. Br Dent J 1996; 180: 329-334.

16 Humphris G M, Morrison T, Lindsay S J E. The Modified Dental Anxiety Scale: validation and United Kingdom norms. Community Dental Health 1995; 12: 143-150.

17 Kent G G, Croucher R. Achieving Oral Health. Oxford: Wright, 1998.

18 Richardson J. Handbook of Qualitative Research Methods for Psychology and the Social Sciences. First edition. Leicester: BPS Books, 1996.

19 Freeman R. Barriers to accessing and accepting dental care. Br Dent J 1999; 187: 81-84.

20 Freeman R. A psychodynamic theory for dental phobia. Br Dent J 1998; 184: 170-172.

21 Milgrom P, Weinstein P, Getz T. Treating Fearful Dental Patients: A Patient Management Handbook. Second edition. Seattle: University of Washington, 1995.

22 Rees L, Lipsedge M, Ball C. Textbook of Psychiatry. Arnold, 1997.

23 Speirs R L. Stress: Physiological Aspects (1). Dent Update 1985; 12: 565572.

24 Jacob M C, Plamping D. The Practice of Primary Dental Care. p 83. Wright: London, 1989.

25 Wardle J. Management of Dental Pain. Paper presented at the British Psychological Society Annual Conference, York, 1982.

26 Kent G, Rubin G, Getz T, Humphris G. Development of a scale to measure the social and psychological effects of severe dental anxiety: social attributes of the Dental Anxiety Scale. Community Dent Oral Epidemiol 1996; 24: 394-397.

27 Jackson C, Lindsay S J E. Reducing anxiety in new dental patients by means of leaflets. Br Dent J 1995; 179: 163-167.

28 Bernstein D, Kleinknecht R, Alexander L. Antecedents of dental fear. J Public Health Dent 1979; 39: 113-124.

29 Millar K, Atkinson J M, Blinkhorn A S, Kay E J. Helping Anxious Adult Patients. Dent Update 1991; 18: 18-25.

30 Segal H. Categories of emergency patient. General Dent 1986; 34: 37-42.

31 Weiner A A. The Difficult Patient: A guide to understanding and managing dental anxiety. 2nd ed. Massachusetts: Reniew Publishing Co., 1994.

32 British Dental Association. Advice Sheet D14 - Violence At Work. BDA Advisory Service, British Dental Association, London, 1997.

33 Locker D, Grushka M. The impact of dental and facial pain. J Dent Res 1987; 66: 1414-1417.

34 Department of Health. An Oral Health Strategy for England. London: Department of Health, 1994.

35 Partridge J. Changing Faces: The challenge of facial disfigurement. 3rd ed. pp 66-67. A Changing Faces Publication, London, 1997.

36 Freeman R. The case for mother in the surgery. Br Dent J 1999; 186: 610613.

37 Crawford A N, Hawker B J, Lennon M A. A dental support group for anxious patients. Br Dent J 1997; 183: 57-62. 